\title{
Hematological Indices of Distant Metastases and Prognostic Nomogram in Gastro-Pancreatic and Biliary Tract Cancers
}

This article was published in the following Dove Press journal: Cancer Management and Research

\author{
Razan Mansour' \\ Osama Abu-Shawer ${ }^{2}$ \\ Ali Lattouf ${ }^{3}$ \\ Hala Sultan ${ }^{3}$ \\ Maysa Al-Hussaini (iD ${ }^{4}$ \\ 'Office of Scientific Affairs and Research, \\ King Hussein Cancer Center, Amman, \\ Jordan; ${ }^{2}$ Department of Internal \\ Medicine, Harvard Medical School, \\ Boston, MA, USA; ${ }^{3}$ School of Medicine, \\ University of Jordan, Amman, Jordan; \\ ${ }^{4}$ Department of Pathology and \\ Laboratory Medicine, King Hussein \\ Cancer Centre, Amman, Jordan
}

Correspondence: Maysa Al-Hussain

Tel +962-6-5300-460//308

Fax +962-6-5300-460//1552

Email mhussaini@khcc.jo
Purpose: The predictive value of inflammatory parameters as indicators of poor overall survival (OS) has been well studied in various tumors. This study aimed to explore the association of neutrophil-lymphocyte ratio (NLR), among other parameters in upper gastrointestinal tract tumors with distant metastases and OS.

Patients and Methods: Retrospective analysis was done for 945 patients (males $n=539$, $57.0 \%$ ) with a median of 57 years (range 19-90 years) treated at King Hussein Cancer Center (KHCC) for gastric $(n=501)$, pancreatic $(n=355)$, and biliary $(n=89)$ carcinoma. NLR, and other parameters were calculated at primary presentation, and the association between the parameters with baseline distant metastases and OS was studied. The optimal cutoff value of NLR was based on receiver operating characteristic curve (ROC) analysis. A prognostic nomogram was then constructed to explore how accurately the OS can be predicted.

Results: Patients with high baseline NLR $(>3.2)$ had more distant metastases on presentation than patients with low NLR $(\leq 3.2)$, (p-value $<0.001)$. Age older than 57 years was associated with poor median OS (8.7 versus 10.6 months, p-value $=0.04)$. In addition, the location of the tumor as stomach versus biliary tract (Odds Ratio $0.44395 \%$ CI $0.281-0.699$ ), and pancreas versus biliary tract (Odds Ratio 1.193, 95\% CI 0.749-1.902, p-value <0.001) proved to be significant. In multivariate analysis, age older than 57 years ( $\mathrm{p}$-value $=0.0033$, HR $0.792,95 \%$ CI $0.678-0.925$ ), location of the tumor ( $\mathrm{p}$-value $<0.0001$ ), presence of distant metastasis (p-value $<0.0001$, HR 2.063, 95\% CI 1.760-2.419), and NLR (p-value $<0.0001$, HR 1.045, 95\% CI $1.028-1.062$ ) remained significant. Nomogram confirmed the significance of NLR as an independent prognostic factor for OS (HR $=1.62,95 \% \mathrm{CI}: 1.31-2.01$, p-value $<0.0001)$.

Conclusion: Our results suggested that high baseline inflammatory markers are associated with distant metastases and poor OS. The utilization of this association in management of cancer patients still warrants further investigation.

Keywords: stomach, pancreas, biliary tract, neutrophil to lymphocyte ratio, nomogram, metastasis

\section{Introduction}

Pancreatic carcinoma, gastric carcinoma, and cholangiocarcinoma are three major malignancies in the upper gastrointestinal tract. ${ }^{1}$ Despite advancement in diagnosis and treatment modalities, the three malignancies are among the most lethal. Pancreatic, gastric and cholangiocarcinoma are associated with 10.1\%, 32.1\% and $19.5 \%$ five year survival rates, respectively. ${ }^{2}$

Evidence is gathering on the roles inflammatory responses might play during different phases of tumor development and progression, ${ }^{3}$ including invasion, ${ }^{4}$ and 
distant metastasis. ${ }^{5}$ This effect extends to the immune surveillance and potential responses to treatment that act as inflammatory mediators, such as tumor necrosis alpha (TNF- $\alpha),{ }^{6}$ along with key pathways involved in inflammation, such as nuclear factor kappa $\mathrm{b}(\mathrm{NF}-\kappa \mathrm{B}){ }^{7}$ and Signal Transducers and Activators of Transcription (STAT3). ${ }^{8}$

The direct mechanism of inflammation leading to metastases is still far from being comprehended. However, there is increasing evidence that inflammation might be among the key determinants of metastases and outcome in cancer patients. These include the absolute neutrophil count (ANC), absolute lymphocyte count (ALC), absolute eosinophil count (AEC), absolute monocyte count (AMC), neutrophil-lymphocyte ratio (NLR), monocyte-lymphocyte ratio (MLR), and plateletlymphocyte ratio (PLR), which were repeatedly described to be associated with a negative impact on survival outcome in various tumors including breast, ${ }^{9}$ non-small cell lung cancer, ${ }^{10}$ testicular tumor, ${ }^{11}$ and tumors of the female genital tract, including ovarian cancers. ${ }^{12}$ Within the gastro-intestinal tract, recent studies showed that elevated NLR appears to be an independent predictive factor for the baseline presence and subsequent development of distant metastases in hepatocellular, ${ }^{13}$ colorectal, $^{14}$ gastric, ${ }^{15}$ and esophageal cancers. $^{16}$

It is hypothesized that inflammatory changes might induce alteration to the microenvironment, hence facilitating tumor promotion, migration and angiogenesis. ${ }^{17}$ Elevated NLR might be related to an increase in circulating neutrophil count and/or the decrease in the lymphocytes count. This imbalance can promote tumor cells' metastasis through the release of various proteases, including VEGF when neutrophils are increased ${ }^{18}$ and the circulating lymphocytes, which are ought to induce cytotoxic tumor-cell death, are decreased. This would eventually lead to a diminished immune response, thus a poor outcome. $^{19}$

Nomograms are not new to the medical field, but their use as predictive tools has become increasingly common in modern medicine. ${ }^{20}$ Nomograms are now utilized in many fields; an important example is oncology, ${ }^{21}$ by incorporating not only tumor characteristics, but other patient specific, and disease determining variables. ${ }^{22}$ Nomograms in oncology were found to have advantages over TNM staging in predicting OS. This is attributed to their ability to be more individualized and personalized, ${ }^{23-25}$ thus pushing towards a more patient-specific approach for management and treatment.

We aim in this retrospective study to further investigate the relationship between blood inflammatory cells primarily with distant metastases and secondarily with OS, among pancreatic, gastric and cholangiocarcinoma. The questions we were trying to address were; how feasible is it to identify reliable and readily available blood inflammatory markers in supporting a decision to select patients who might benefit from a more active surveillance for the detection of early metastasis before it is clinically apparent? Can we confirm the previously reported effect of high NLR on poor OS? And can these markers help in triaging patients for both current and developing treatments such as immunotherapy?

\section{Materials and Methods}

\section{Patients}

This is a retrospective chart review study approved by the Institutional Review Board (IRB) office at King Hussein Cancer Center (KHCC). The IRB at KHCC works in accordance with the Declaration of Helsinki to protect the rights and welfare of all research participants. The informed consent is waivered under the bylaws of the KHCC-IRB if there will be retrospective use of data collected for non-research purposes. The identity of the participants remains unidentified during the collection of and later on the analysis of the data. The records of 945 patients with gastric $(n=501)$, pancreatic $(n=355)$, and biliary $(n=89)$ carcinoma who received their treatment at $\mathrm{KHCC}$ were reviewed. The reports of Positron emission tomography (PET), computed tomography (CT), and magnetic resonance imaging (MRI) scans were reviewed to detect the presence of baseline distant metastases. Clinical data including age, gender, and sites of baseline distant metastases are all summarized in Table 1. We excluded all patients who were documented to be on steroid therapy, had current infection, or underwent surgery before obtaining their first complete blood count (CBC) at KHCC. Using steroids was expected to confound the results of the study as it leads to leukocytosis, more specifically neutrophilia. $^{16,17}$

CBC with differential white cell count was collected before the initiation of any cancer-specific treatment (systemic treatment or radiation). The pre-treatment baseline NLR, MLR and PLR were calculated using these formulas; $\mathrm{NLR}=\mathrm{ANC} /$ $\mathrm{ALC}, \mathrm{MLR}=\mathrm{AMC} / \mathrm{ALC}$ and $\mathrm{PLR}=$ Platelet Count $/$ ALC.

\section{Analysis}

The Receiver Operating Characteristic (ROC) curve was operated to determine the best NLR cut-off value for the 
Table I Demographic and Clinical Findings in Patients with Gastric, Pancreatic and Cholangiocarcinoma

\begin{tabular}{|c|c|c|}
\hline Variable & Value & Number (\%) \\
\hline \multirow[t]{2}{*}{ Gender } & Females & 406 (43.0\%) \\
\hline & Males & 539 (57.0\%) \\
\hline \multirow[t]{2}{*}{ Age group (years) } & $>57$ & 457 (48.4\%) \\
\hline & $\leq 57$ & 488 (51.6\%) \\
\hline \multirow[t]{3}{*}{ Location } & Stomach & $50 \mid$ (53.0\%) \\
\hline & Pancreas & 355 (37.6\%) \\
\hline & Biliary tract & 89 (9.4\%) \\
\hline \multirow[t]{2}{*}{ Any metastasis } & No & $529(56.0 \%)$ \\
\hline & Yes & 416 (44.0\%) \\
\hline \multirow[t]{3}{*}{ Liver metastasis } & NA & 33 \\
\hline & No & 644 (70.6\%) \\
\hline & Yes & 268 (29.4\%) \\
\hline \multirow[t]{3}{*}{ Peritoneal metastasis } & NA & 33 \\
\hline & No & 787 (86.3\%) \\
\hline & Yes & $125(13.7 \%)$ \\
\hline \multirow[t]{3}{*}{ Lung metastasis } & NA & 34 \\
\hline & No & 825 (90.6\%) \\
\hline & Yes & $86(9.4 \%)$ \\
\hline \multirow[t]{3}{*}{ Bone metastasis } & NA & 59 \\
\hline & No & 825 (93.1\%) \\
\hline & Yes & 61 (6.9\%) \\
\hline \multirow[t]{2}{*}{ Patient status } & Alive & $284(30.1 \%)$ \\
\hline & Dead & 661 (69.9\%) \\
\hline
\end{tabular}

Abbreviation: NA, not available.

association with the presence of baseline distant metastases collectively from the three sites, matching the most extreme joint sensitivity and specificity. The association between NLR, age, gender, and the location of the tumor (stomach, pancreas, and biliary tract including intra- and extrahepatic) with the presence of baseline distant metastases was tested. Univariate and multivariate logistic regression analyses were used to examine the association between the various tested variables and the presence of baseline distant metastases. A p-value of $\leq 0.05$ was determined as the cutoff value for significance association.

Our analysis proceeded stepwise. In the first phase, we examined the association between the baseline NLR with the presence of distant metastases. In the second phase, we examined the association between other hematologic parameters including ANC, ALC, AEC, AMC, MLR and PLR with the baseline presence of distant metastases. In the third phase, we examined the association between the baseline presence of distant metastases with clinical variables like age, gender, and location of the tumor. In the fourth phase, we performed a multivariate analysis that included the collected variables including the age, gender, median NLR and location. In the fifth phase, we examined the association between the hematologic parameters including ANC, ALC, AEC, AMC, NLR, MLR and PLR with the OS.

Descriptive analysis of patients' information was done. Categorical data, such as age group, gender, and other factors were presented as counts and percentages. The median, mean, standard deviation and range were calculated for the continuous data including age, and the hematological parameters. In general, differences in proportions were tested with $\chi^{2}$ test or Fisher's exact test, and differences in continuous variables were tested with Student's $t$-test or nonparametric test (Willxon Rank Test) depending on the assumptions required for each test. Multivariate analysis included gender, age, location of the tumor (stomach, pancreas, cholangiocarcinoma), and NLR cutoff (NLR $>3.2$ vs $\leq 3.2$ ) with probability modeled on (stage ="IV"), using a logistic regression model. The Kaplan-Meier method was used to estimate OS curves, and the Log rank test was used to compare patients' survival times between factors' groups. The OS time was calculated from the date of diagnosis up until the date of death from any cause. Survival was expressed as median with a $95 \%$ confidence interval. Multivariate analysis was done using Cox proportional hazards regression model. ROC curve was done for NLR, with stage and OS. A significance criterion of $p$-value $\leq 0.05$ was used in the analysis. All analyses were performed using SAS version 9.4 (SAS Institute Inc, Cary, NC).

\section{Developing a Prognostic Nomogram}

In addition, an attempt to develop a predictive nomogram based on available variables was undertaken. For this purpose, the analysis was redone only on cases without any missing variables $(n=839)$. The sample was then divided into a training group (two-thirds from all locations; $\mathrm{n}=587$, including gastric $n=281$, pancreatic $n=245$, and cholangiocarcinoma $\mathrm{n}=61$ ), and a validation group (the remaining onethird from all locations $n=252$, including gastric $n=121$, pancreatic $\mathrm{n}=105$, and cholangiocarcinoma $\mathrm{n}=26$ ). Patients were shuffled then randomly selected into $70 \%$ for the training set and $30 \%$ for the validation set. R-software was used for the analysis and for building up the nomogram. For the training group the ROC represents the "Area under the 
ROC Curve" (AUC) which was calculated at every half-year intervals from the first to the fifth year, the solid line represents the mean of the AUC, and the dashed line represents the median of the AUC. The darker interval in the plot shows the $25 \%$ and $75 \%$ quantiles of $\mathrm{AUC}$ and the lighter interval shows the minimum and maximum of AUC (Supplementary Figure 1A). The ROC-for the validation group shows the AUC on the validation dataset (Supplementary Figure 1B).

Calibration is utilized to measure how accurate the model predictions are from the actual OS. The KaplanMeier method was used to draw the survival curves in relation to the risk stratification. The Nomogram was then developed for the training group.

\section{Results}

There were 945 cases in the three locations. The median age at diagnosis was 57 years (range 19-90 years). More than half of the patients were males $(57 \%)$, with a male to female ratio of 1.3:1. The median OS for all patients was 9.6 months. The median baseline white blood count (WBC) was 8.1 per $\mu \mathrm{L}$ (mean 9.174, range 1.9-82.7). The median baseline NLR was 3.29 , and the mean baseline NLR was 4.37. The clinical characteristics of 945 patients with upper gastrointestinal tract cancer and the $\mathrm{CBC}$ counts including the various white blood cells and platelet absolute counts, along with the hematologic parameters' ratios are summarized in Tables 1 and 2, respectively.

The cutoff value of NLR for the association with baseline distant metastases was 3.2, determined using the ROC curve where the AUC was 0.5945 (Supplementary Figure 2).

The relationship between the variables including the age, gender and tumor location, as well as the peripheral count of the various immune cells and their ratios with the baseline presence of distant metastases is summarized in Table 3. Patients with elevated baseline NLR $(>3.2)$ were more likely to have distant metastases at time of presentation in comparison to patients with low baseline NLR $(\leq 3.2)$, (p-value $<0.001$, Odds Ratio (OR): 1.954, CI: 1.505-2.539). Furthermore, patients with high baseline ANC (median $>5730$ / $\mu \mathrm{L}, \mathrm{OR} 1.596,95 \%$ CI 1.232-2.069), MLR (median $>0.33 / \mu \mathrm{L}$, OR 1.496, 95\% CI 1.155-1.939), and PLR ( $\geq 0.154$, OR 1.318, 95\% CI 1.014-1.712) had more distant metastases in comparison to patients with low baseline ANC $(\leq 5730 / \mu \mathrm{L})$, MLR $(\leq 0.33 / \mu \mathrm{L})$, and PLR $(\leq 0.154)$ (p-value $0.000,0.002$, and 0.039 , respectively). AEC was not associated with the presence of baseline distant metastases ( $p$-value $=0.471$ ).

Multivariate analysis was utilized for testing the association of clinical variables, the primary location of the tumor and NLR with baseline distant metastases. Despite insignificant association in univariate analysis, both gender and age group were included in the multivariate analysis as these are deemed important clinical determinants of outcome. Age, but not gender, along with NLR and tumor location were associated significantly with the baseline presence of distant metastases. Table 4 shows the univariate and multivariate analysis of the age, gender, NLR and location of the tumor with the presence of baseline distant metastasis at the time of presentation.

For the survival analysis, the median OS for the whole group was 9.6 months (Figure 1). Age older than 57 years was associated with poor median OS (8.7 versus 10.6 months, HR 0.851, 95\% CI 0.730-0.993, p-value 0.04) (Figure 2). Based on tumor location, pancreatic carcinoma had the worst OS (6.6 months, HR pancreatic versus biliary tract 1.514 , 95\% CI 1.148-1.997) followed by cholangiocarcinoma and gastric carcinoma (11 months, and 12.3 months, respectively, HR gastric versus biliary 0.748 , 95\% CI 0.566-0.989), as shown in Figure 3.

Table 2 The Peripheral Count of Various Immune Cells in This Group of Patients

\begin{tabular}{|l|l|l|l|l|l|l|}
\hline Variable & Median & Range & Max & Min & Std & Mean \\
\hline ANC & 5730 & 184,978 & 185,248 & 270 & 12,332 & 9214.38 \\
ALC & 1892 & 18,196 & 18,360 & 164 & 1851 & 2383.54 \\
AMC & 634.4 & 74,430 & 74,430 & 0 & 2473 & 876.59 \\
AEC & 171.8 & 18,900 & 18,900 & 0 & 726 & 282.85 \\
NLR->ANC/ALC & 3.29 & 46.93 & 47.05 & 0.122 & 4 & 4.37 \\
MLR->AMC/ALC & 0.33 & 10 & 10 & 0 & 0 & 0.411 \\
PLR->PLT/ALC & 0.154 & 1.437 & 1.437 & 0 & 0 & 0.181 \\
Platelets & 275 & 1354 & 1354 & 0 & 167 & 285.46 \\
\hline
\end{tabular}

Abbreviations: AEC, absolute eosinophil count; ALC, absolute lymphocyte count; AMC, absolute monocyte count; ANC, absolute neutrophil count; MLR, monocytelymphocyte ratio; NLR, neutrophil-lymphocyte ratio; PLR, platelet-lymphocyte ratio; PLT, platelets; Std, standard deviation. 
Table 3 The Association Between the Various Variables Including Hematologic Indices with the Presence of Baseline Distant Metastases

\begin{tabular}{|c|c|c|c|c|c|}
\hline \multirow[t]{2}{*}{ Variable } & \multirow[t]{2}{*}{ Value } & \multirow[t]{2}{*}{ Total } & \multicolumn{2}{|l|}{ Metastasis } & \multirow[t]{2}{*}{ p-value } \\
\hline & & & Yes & No & \\
\hline \multirow{2}{*}{$\begin{array}{l}\text { Age group } \\
\text { (Years) }\end{array}$} & Age $>57$ & 457 & $190(41.6 \%)$ & $267(58.4 \%)$ & 0.143 \\
\hline & Age $\leq 57$ & 488 & $226(46.3 \%)$ & $262(53.7 \%)$ & \\
\hline \multirow[t]{2}{*}{ Gender } & Female & 406 & $180(44.3 \%)$ & $226(55.7 \%)$ & 0.866 \\
\hline & Male & 539 & $236(43.8 \%)$ & $303(56.2 \%)$ & \\
\hline \multirow[t]{3}{*}{ Location } & BT & 89 & $47(52.8 \%)$ & $42(47.2 \%)$ & $<0.001$ \\
\hline & Stomach & 501 & $166(33.1 \%)$ & 335 (66.9\%) & \\
\hline & Pancreas & 355 & $203(57.2 \%)$ & $152(42.8 \%)$ & \\
\hline \multirow[t]{3}{*}{ ANC median } & NA & 3 & $2(. \%)$ & I (. \%) & $<0.001$ \\
\hline & ANC $\leq 5730$ & 471 & $180(38.2 \%)$ & 291 (61.8\%) & \\
\hline & ANC $>5730$ & 471 & $234(49.7 \%)$ & $237(50.3 \%)$ & \\
\hline \multirow[t]{3}{*}{ ANC mean } & NA & 3 & $2(. \%)$ & I (. \%) & 0.036 \\
\hline & ANC $\leq 9214.38$ & 716 & $301(42.0 \%)$ & $415(58.0 \%)$ & \\
\hline & ANC $>9214.38$ & 226 & $113(50.0 \%)$ & $113(50.0 \%)$ & \\
\hline \multirow[t]{3}{*}{ AMC median } & NA & 4 & $2(. \%)$ & $2(. \%)$ & 0.141 \\
\hline & AMC $\leq 634.4$ & 471 & $196(41.6 \%)$ & 275 (58.4\%) & \\
\hline & AMC $>634.4$ & 470 & $218(46.4 \%)$ & $252(53.6 \%)$ & \\
\hline \multirow[t]{3}{*}{ AMC mean } & NA & 4 & $2(. \%)$ & $2(. \%)$ & 0.477 \\
\hline & AMC $\leq 876.59$ & 686 & $297(43.3 \%)$ & 389 (56.7\%) & \\
\hline & AMC $>876.59$ & 255 & 117 (45.9\%) & 138 (54.1\%) & \\
\hline \multirow[t]{3}{*}{ ALC median } & NA & 3 & $2(. \%)$ & I (. \%) & 0.056 \\
\hline & ALC $\leq 1892$ & 472 & $222(47.0 \%)$ & $250(53.0 \%)$ & \\
\hline & ALC $>1892$ & 470 & $192(40.9 \%)$ & 278 (59.1\%) & \\
\hline \multirow[t]{3}{*}{ ALC mean } & NA & 3 & $2(. \%)$ & I (. \%) & 0.019 \\
\hline & ALC $\leq 2383.54$ & 640 & 298 (46.6\%) & $342(53.4 \%)$ & \\
\hline & ALC $>2383.54$ & 302 & $116(38.4 \%)$ & $186(61.6 \%)$ & \\
\hline \multirow[t]{3}{*}{ AEC median } & NA & 163 & $74(. \%)$ & $89(. \%)$ & 0.471 \\
\hline & $A E C \leq 171.8$ & 391 & $166(42.5 \%)$ & 225 (57.5\%) & \\
\hline & $A E C>171.8$ & 391 & $176(45.0 \%)$ & $215(55.0 \%)$ & \\
\hline \multirow[t]{3}{*}{ AEC mean } & NA & 163 & $74(. \%)$ & $89(. \%)$ & 0.893 \\
\hline & $\mathrm{AEC} \leq 282.85$ & 553 & $24 \mid(43.6 \%)$ & $312(56.4 \%)$ & \\
\hline & $\mathrm{AEC}>282.85$ & 229 & $101(44.1 \%)$ & $128(55.9 \%)$ & \\
\hline \multirow[t]{3}{*}{ NLR median } & NA & 3 & $2(. \%)$ & I (. \%) & $<0.001$ \\
\hline & $N L R \leq 3.29$ & 470 & $170(36.2 \%)$ & 300 (63.8\%) & \\
\hline & NLR $>3.29$ & 472 & 244 (51.7\%) & $228(48.3 \%)$ & \\
\hline \multirow[t]{3}{*}{ NLR mean } & NA & 3 & $2(. \%)$ & I (. \%) & $<0.001$ \\
\hline & $N L R \leq 4.37$ & 637 & $251(39.4 \%)$ & $386(60.6 \%)$ & \\
\hline & $N L R>4.37$ & 305 & $163(53.4 \%)$ & I 42 (46.6\%) & \\
\hline \multirow[t]{3}{*}{ NLR cutoff } & NA & 3 & $2(. \%)$ & I (. \%) & $<0.001$ \\
\hline & $N L R \leq 3.2$ & 456 & $162(35.5 \%)$ & $294(64.5 \%)$ & \\
\hline & $N L R>3.2$ & 486 & 252 (51.9\%) & 234 (48.1\%) & \\
\hline
\end{tabular}

Abbreviations: AEC, absolute eosinophil count; ALC, absolute lymphocyte count; AMC, absolute monocyte count; ANC, absolute neutrophil count; BT, biliary tract; NA, not available; NLR, neutrophil lymphocyte ratio.
Furthermore, those with distant metastasis had a significantly lower survival than those without (p-value $<0.0001$, HR 2.072, 95\% CI 1.771-2.423). For the hematological variables; ANC (median ANC $>5730$, HR 1.409, 95\% CI 1.207-1.643), NLR (median NLR $>3.29$, HR $1.611,95 \%$ CI $1.380-1.880$ ), MLR (median MLR $>0.33$, HR 1.424, 95\% CI 1.220-1.662) and PLR (median PLR $>0.154$, HR 1.414, 95\% CI 1.210-1.653) were associated with poor OS (p-value $<0.0001,<0.0001,0.0001$, and $<0.0001$, respectively). Supplementary Figure $3 \mathrm{~A}-\mathrm{E}$ shows the graphs for the associations. In addition, multivariate cox regression was utilized to determine the remaining significant variables when co-founded by others. Age $>57$ years, primary location of the tumor, the presence of distant metastasis and the NLR as a continuous variable remained significant, as shown in Table 5.

Analysis of hematological indices was then performed for each type of malignancy to determine their significance in specific types of tumors. In 355 pancreatic cancer patients, analyses revealed that patients with high NLR ( $\geq 3.3$ ) had more baseline distant metastases compared to patients with low NLR (<3.3), (p-value: <0.0001) . Patients with high baseline ANC $(\geq 5500 / \mu \mathrm{L})$, AMC $(\geq 600 / \mu \mathrm{L})$, MLR $(\geq 0.3)$ had more baseline distant metastases in comparison to patients with low baseline ANC $(<5500 / \mu \mathrm{L}), \mathrm{AMC}(<600 / \mu \mathrm{L})$, and MLR $(<0.3)$, (p-value: $0.02,0.001$, and $<0.0001$, respectively) (Supplementary Table 1). High ANC, NLR, MLR, and PLR and low ALC were associated with poorer OS (p-value: $<0.0001$, $<0.0001,<0.0001,0.04$, and 0.01 , respectively). In 501 gastric cancer patients, high baseline NLR (>3.29) patients had more distant metastases on presentation than low baseline NLR ( $\leq 3.29)$ patients (p-value: 0.003). Moreover, patients with high baseline ANC (>5730), and PLR $(>0.15)$ had more distant metastases in comparison to patients with low baseline ANC $(\leq 5730)$ and PLR $(\leq 0.15) \quad$ (p-value: 0.012 , and 0.022 , respectively) (Supplementary Table 2). OS was found to be poor in patients with high NLR, ANC, MLR and PLR (p-value: $0.0003,0.046,0.023$, and 0.0001 , respectively). In 89 patients with biliary tract cancer, high levels of baseline NLR (>3.29), ANC (>5730), and ALC (>1892) were not associated with the presence of baseline distant metastasis more than patients with low NLR $(\leq 3.29)$, ANC $(\leq 5730)$, and ALC ( $\leq 1892$ ), (p-value: 0.357, 0.370, and 0.776, respectively) (Supplementary Table 3). Patients with high 
Table 4 Multivariate Analyses for the Association of the Different Variables with the Presence of Baseline Distant Metastases

\begin{tabular}{|l|l|l|l|l|}
\hline & $\begin{array}{l}\text { Odds } \\
\text { Ratio }\end{array}$ & \multicolumn{2}{|l|}{$\begin{array}{l}\text { 95\% Wald } \\
\text { Confidence } \\
\text { Limits }\end{array}$} & p-value \\
\hline Gender (male vs female) & 0.964 & 0.734 & 1.267 & 0.7949 \\
Age group (age $\leq 57$ vs age $>57$ ) & 1.545 & 1.171 & 2.039 & 0.0021 \\
NLR cutoff (NLR>3.2 vs NLR $\leq 3.2)$ & 2.140 & 1.627 & 2.815 & $<0.0001$ \\
Location (Stomach vs BT) & 0.405 & 0.253 & 0.648 & $<0.0001$ \\
Location (Pancreas vs BT) & 1.218 & 0.753 & 1.969 & \\
\hline
\end{tabular}

Abbreviations: $B T$, biliary tract; NLR, neutrophil-lymphocyte ratio.

baseline NLR ( $>3.29)$ had a median OS of 14.07 months compared to patients with low NLR $(\leq 3.29)$, who had a median survival of 9.475 months ( $p$-value: 0.7075 ).

\section{Developing the Predictive Nomogram Model}

A total of 839 cases (including $n=402$ gastric, $n=350$ pancreatic, and $\mathrm{n}=87$ for cholangiocarcinoma) were included and were divided into two-thirds training group and one-third validation group as described above. There was no significant difference between the training and the validation groups in terms of the gender, median age, location of the tumor, and stage at diagnosis. In addition, the training and validation groups were both homogenous in terms of follow-up duration, NLR, MLR, and PLR. The median OS for the training group was 9.57 months (95\% CI 8.660-11.0), with the survival rate at 1,3 , and 5 years of $43.4 \%, 19.6 \%$, and $14.3 \%$, respectively. For the validation group, the median OS was 10.0 months, with a survival rate at 1,3 , and 5 years of $42.6 \%, 19.6 \%$, and $15.4 \%$, respectively. (Supplementary Figure 4A and B).

For the training group, univariate analysis showed that cancer patients with MLR $>0.3, \quad$ NLR $>3.1$, PLR $>0.1$, ALC $>1890$ and ANC $>5698$ scores had poor prognosis (p-values $0.0032,<0.0001,0.0324,0.0018$ and $<0.0001$, respectively). Multivariate analysis further revealed that NLR (HR $=1.62,95 \%$ CI: $1.31--$ 2.01, p-value $<0.0001$ ), tumor location, and distant metastases were independent risk factors for prognosis and poor OS ( $\mathrm{p}$-value $<0.0001$, and $<0.0001$, respectively), but MLR and PLR showed no significant influence on prognosis (p-value 0.5218 and 0.7436 , respectively). Tables 6 and 7 .

All variables were included in the construction of the nomogram for prediction of the 5-year survival in these patients based on the NLR group (NLR group 0 means NLR $\leq 3.1$, and NLR group 1 means NLR>3.1), and distant metastasis at the time of presentation (Figure 4).

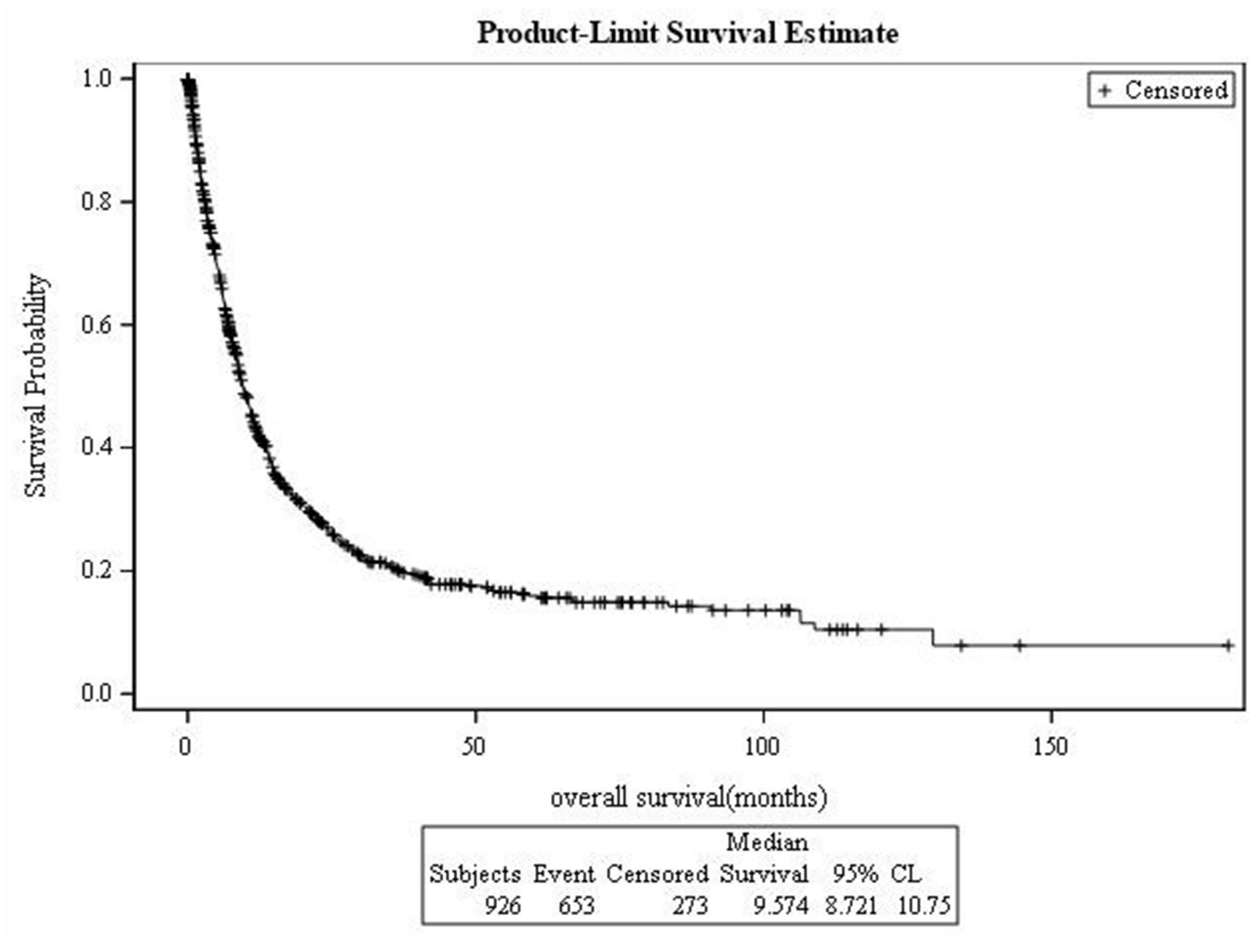

Figure I Kaplan-Meier curve of overall survival for the whole group. 


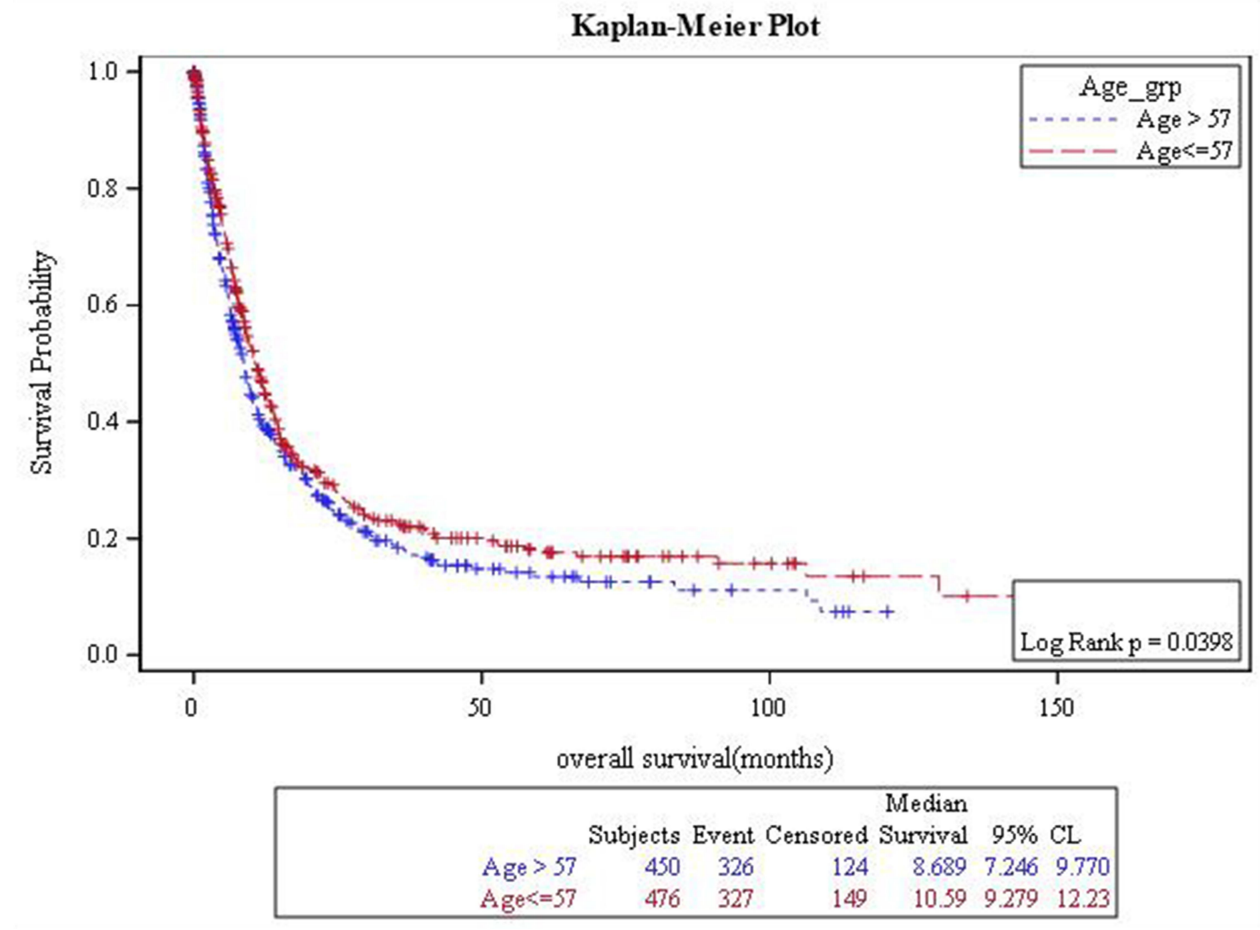

Figure 2 Kaplan-Meier curve of overall survival based on median age.

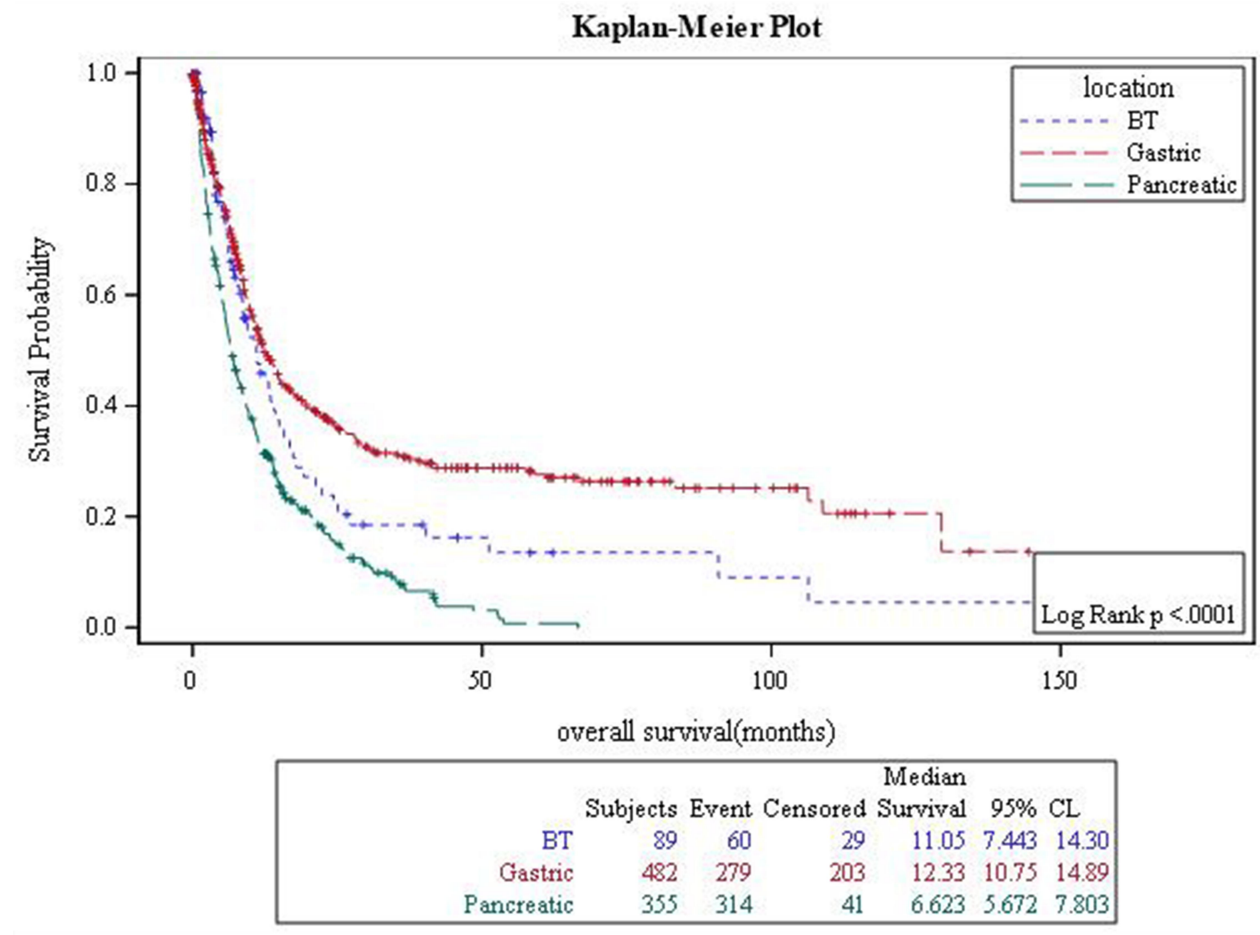

Figure 3 Kaplan-Meier curve of overall survival for patients based on location of the tumor.

The sum of the points at the top of the scale for each factor was first measured, and was then assessed on the lower total points scale to determine the 5-year OS probability.
Furthermore, calibration aimed at measuring how far the model predictions were from the actual survival outcomes for both the training and validation groups. This showed that the 
Table 5 Multivariate Analysis for the OS in Gastric, Pancreatic and Cholangiocarcinoma

\begin{tabular}{|l|l|l|l|l|l|}
\hline Parameter & & p-value & Hazard Ratio & \multicolumn{2}{|l|}{$\mathbf{9 5 \%}$ Hazard Ratio Confidence Limits } \\
\hline Metastasis & Yes vs No & $<0.0001$ & 1.873 & 1.594 & 2.200 \\
NLR & By unit & $<0.0001$ & 1.048 & 1.032 & 1.065 \\
Age group & Age $\leq 57$ vs Age $>57$ & 0.0431 & 0.851 & 0.728 & 0.995 \\
Sex & Male vs Female & 0.2869 & 0.919 & 0.786 & 1.074 \\
Location & Stomach vs BT & $<0.0001$ & 0.890 & 0.670 & 1.181 \\
& Pancreas vs BT & & 1.603 & 1.210 & 2.124 \\
\hline
\end{tabular}

Abbreviations: BT, biliary tract; NLR, neutrophil-lymphocyte ratio; OS, overall survival.

Table 6 Univariate and Multivariate Cox Regression Analysis on Factors Affecting the OS in the Training Group

\begin{tabular}{|c|c|c|c|c|}
\hline Parameter & & HR $(95 \% \mathrm{Cl})$ & p-value & Adjusted $\mathrm{p}$-value \\
\hline Age & Age $>57$ vs Age $\leq 57$ & $1.16,(0.96,1.41)$ & 0.1345 & 0.1345 \\
\hline Gender & Female vs Male & I.18,(0.97, I.43) & 0.1000 & 0.1000 \\
\hline Location & $\begin{array}{l}\text { Stomach vs.BT } \\
\text { Pancreas vs BT }\end{array}$ & $\begin{array}{l}0.81,(0.57,1.15) \\
1.76,(1.25,2.48)\end{array}$ & $\begin{array}{l}0.2303 \\
0.0011\end{array}$ & $<0.0001$ \\
\hline Metastasis (stage) & $\begin{array}{l}\text { Yes vs No } \\
\text { (IV vs I-III) }\end{array}$ & $2.06,(1.69,2.51)$ & $<0.0001$ & $<0.0001$ \\
\hline Liver metastasis & Yes vs No & $2.33,(1.89,2.87)$ & $<0.0001$ & $<0.0001$ \\
\hline Lung metastasis & Yes Vs No & $1.94,(1.44,2.62)$ & $<0.0001$ & $<0.0001$ \\
\hline Bone met-astasis & Yes vs No & I.23,(0.87, I.73) & 0.2456 & 0.2456 \\
\hline Peritoneal metastasis & Yes vs No & $1.57,(1.19,2.07)$ & 0.0012 & 0.0012 \\
\hline WBC grp & $W B C>8.3$ vs $W B C \leq 8.3$ & $1.4 I,(1.16,1.7 I)$ & 0.0005 & 0.0005 \\
\hline ANC grp & ANC $>5698$ vs ANC $\leq 5698$ & $1.49,(1.23,1.80)$ & $<0.0001$ & $<0.0001$ \\
\hline ALC grp & $A L C>1890$ vs $A L C \leq 1890$ & $0.74,(0.61,0.89)$ & 0.0018 & 0.0018 \\
\hline AMC grp & $A M C>616$ vs $A M C \leq 616$ & $0.97,(0.80,1.17)$ & 0.7449 & 0.7449 \\
\hline ANC grp & ANC $>5698$ vs ANC $\leq 5698$ & $1.49,(1.23,1.80)$ & $<0.0001$ & $<0.0001$ \\
\hline NLR grp & $\begin{array}{l}\text { NLR median }>3.1 \\
\text { Vs NLR } \leq 3.1\end{array}$ & $1.73,(1.42,2.10)$ & $<0.0001$ & $<0.0001$ \\
\hline MLR grp & $M L R>0.3$ vs $M L R \leq .3$ & $1.34,(1.10,1.63)$ & 0.0032 & 0.0032 \\
\hline PLR grp & $P L R>0.1$ vs $P L R \leq 0.1$ & $1.29,(1.02,1.62)$ & 0.0324 & 0.0324 \\
\hline
\end{tabular}

Abbreviations: AEC, absolute eosinophil count; ALC, absolute lymphocyte count; AMC, absolute monocyte count; ANC, absolute neutrophil count; BT, biliary tract; $\mathrm{Cl}$, confidence interval; grp, group; HR, hazard ratio; NLR, neutrophil to lymphocyte ratio; MLR, monocyte to lymphocyte ratio; PLR, platelet to lymphocyte ratio; PLT, platelets; WBC, white cell count.

predictive height of the nomogram was consistent with the actual observations of patients' survival (Figure 5A and B), and could stratify the patients into low, intermediate, and high-risk categories. The calibration chart of the training group showed that the 5-year survival rates predicted by the nomogram were consistent with the actual observation in the high risk (95\% CI 0.03721771-0.1459379), intermediate risk (95\% CI $0.05930160-0.1896795)$, and low risk (95\% CI $0.18166932-0.3322087$ ) categories.

The AUC of the nomogram in both the training and validation cohort showed that the median and the mean are in close proximity in each sample, indicating that the model was stable and valid. The ROC and AUC were done on the training group for the NLR, PLR, and MLR for the OS. NLR constantly maintained a higher value, indicating the superiority of NLR over other inflammatory markers in predicting OS in those patients.

Kaplan-Meier survival analysis in the training and validation cohorts showed that cancer patients among the highrisk category were statistically associated with the risk of a lower OS probability compared to the groups of patients in the intermediate and low risks categories ( $p$-value $<0.001$ ). (Figure 6A and B)

\section{Discussion}

In this retrospective study, we examined the association of NLR, ANC, ALC, AEC, AMC, MLR and PLR with the presence of baseline distant metastases and OS in 945 
Table 7 Multivariate Cox Regression Analysis on Factors Affecting the OS in the Training Group

\begin{tabular}{|c|c|c|c|}
\hline Parameter & $\begin{array}{l}\text { Adjusted HR (95\% } \\
\mathrm{Cl})\end{array}$ & p-value & $\begin{array}{l}\text { Adjusted } \\
\text { p-value }\end{array}$ \\
\hline Location & $\begin{array}{l}\text { Stomach vs. BT } 0.86 \\
(0.60,1.22) \\
\text { Pancreas vs. BT I.7I } \\
(1.21,2.40)\end{array}$ & $\begin{array}{l}0.3832 \\
0.0022\end{array}$ & $<0.0001$ \\
\hline Metastasis & I.78 $(1.46,2.18)$ & $<0.0001$ & $<0.0001$ \\
\hline NLR median group & $1.62(1.31,2.01)$ & $<0.0001$ & $<0.0001$ \\
\hline MLR group & $\mathrm{I} .07(0.86, \mathrm{I} .33)$ & 0.5218 & 0.5218 \\
\hline PLR group & $1.04(0.82,1.33)$ & 0.7436 & 0.7436 \\
\hline
\end{tabular}

Abbreviations: $\mathrm{BT}$, biliary tract; $\mathrm{Cl}$, confidence interval; $\mathrm{HR}$, hazard ratio; MLR, monocyte-lymphocyte ratio; NLR, neutrophil-lymphocyte ratio; OS, overall survival; PLR, platelet-lymphocyte ratio.

patients with pancreatic, gastric, and biliary tract carcinomas. We also challenged the predictive potential of using NLR as a novel marker for detecting distant metastases in those patients. An elevated baseline NLR $(>3.2)$ was an independent factor associated with baseline presence of distant metastases in gastric, pancreatic and biliary tract cancer patients collectively, as depicted in univariate and multivariate analyses, after adjusting for important covariates such as location of the tumor and age (p-value $<0.0001$, and $\mathrm{p}$-value $=0.0021$, respectively $)($ Table 4$)$. Also, patients with high baseline ANC $(>5730 / \mu \mathrm{L})$, MLR $(>0.33)$, and PLR $(>0.154)$ had more distant metastases in comparison to patients with low baseline ANC $(\leq 5730 /$ $\mu \mathrm{L})$, MLR $(\leq 0.33 / \mu \mathrm{L})$, and PLR $(\leq 0.154)$ (p-value $<0.001$, 0.002 , and 0.039 , respectively).
Compared with the cut-off NLR values determined through published and unpublished literature for pancreatic, gastric, and cholangiocarcinoma separately (3.3, 3.31, and 3.17 , respectively), the ROC curve analysis in this study was plotted to identify an optimal NLR cut-off value for the association with the presence of baseline distant metastases from these three sites collectively. This combined NLR value of 3.2 for all three locations ensured attaining the most extreme joint sensitivity and specificity.

We have also constructed a prognostic nomogram based on NLR and the presence or absence of distant metastases on 839 patients. Analysis confirmed that elevated NLR ( $>3.1)$ was associated with poor prognosis of pancreatic, gastric, and biliary tract cancer patients in the training $(\mathrm{n}=587)$ and validation $(\mathrm{n}=252)$ groups. NLR remained an independent prognostic factor for these patients. NLR $>3.1$ versus $\leq 3.1$ was a significant predictor of worst OS at 1,3 , and 5 years $(30.9 \%$ vs. $55.0 \%, 13.53 \%$ vs $25.28 \%$ and $12.09 \%$ versus $16.4 \%$, respectively)

Many studies have shed a light on tumor-associated inflammation, and increasingly on inflammatory markers as determinants of progression and OS in patients with cancer, ${ }^{26,27}$ in which NLR as well as other inflammatory markers were associated with poor prognosis and OS. The relationship between inflammation and cancer has been well established in the literature. ${ }^{28}$ Neutrophils can produce various chemokines and cytokines that help further suppress immunity. Neutrophils have been assumed as important mediators in cancer progression because they promote tumor growth through cytokine release, stimulate

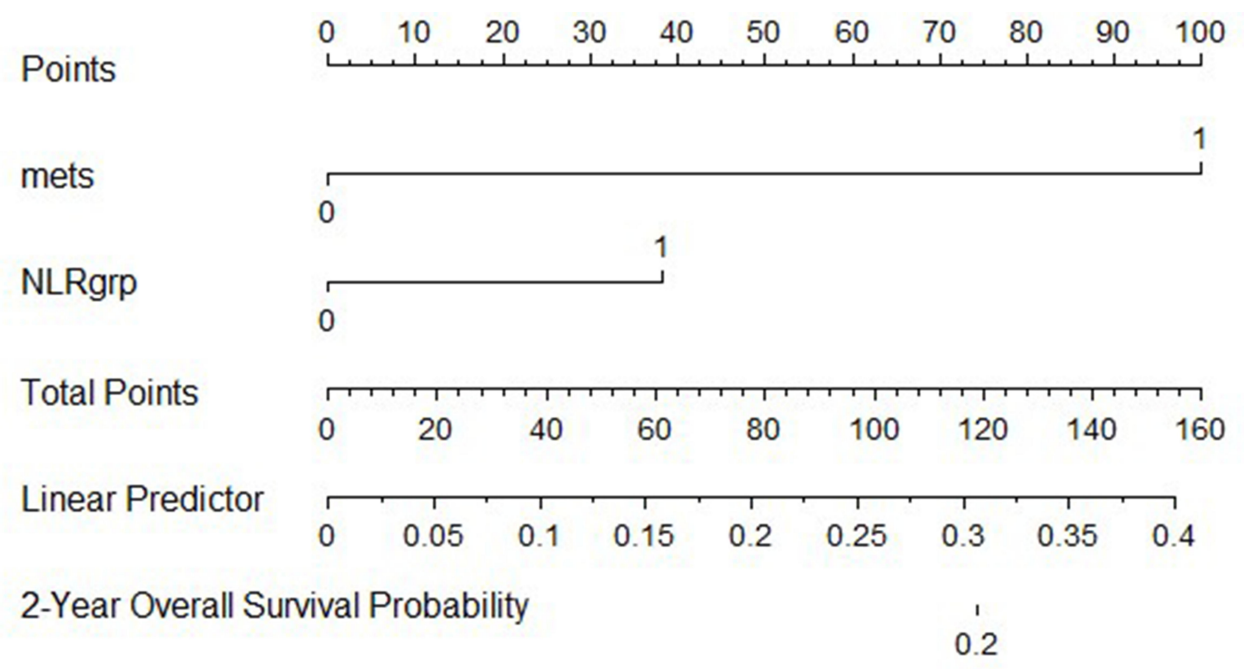

Figure 4 The constructed nomogram depending on the significant variable. 

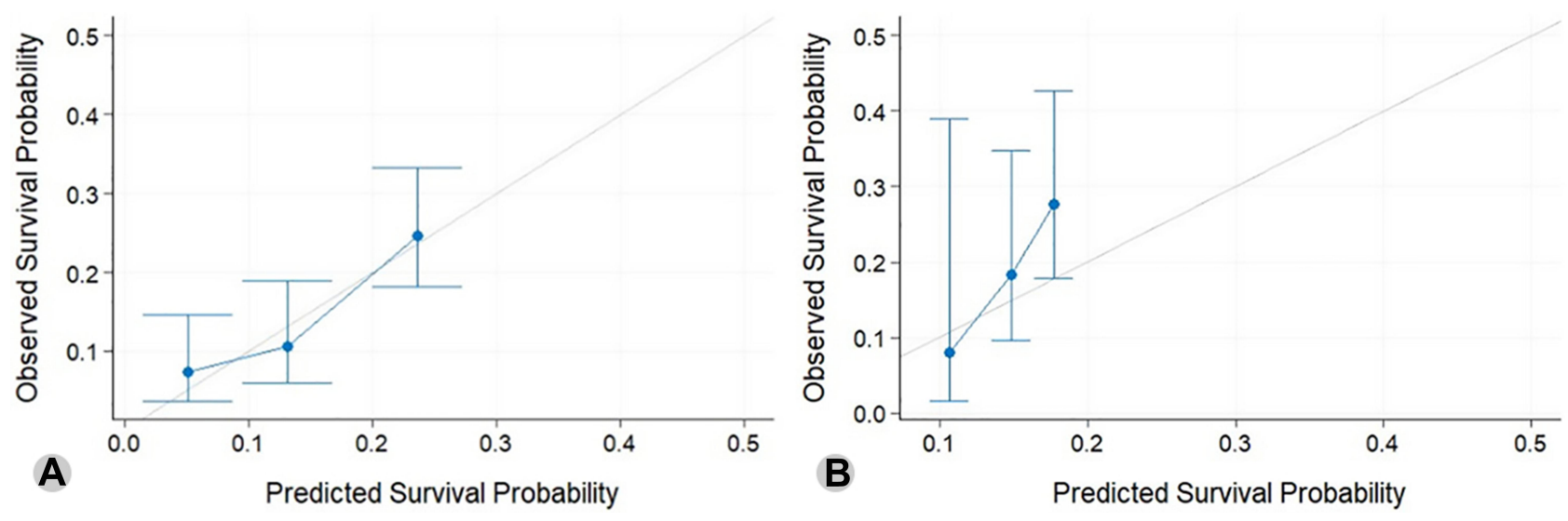

Figure 5 Calibration curves for the training $(\mathbf{A})$, and validation $(\mathbf{B})$ groups.
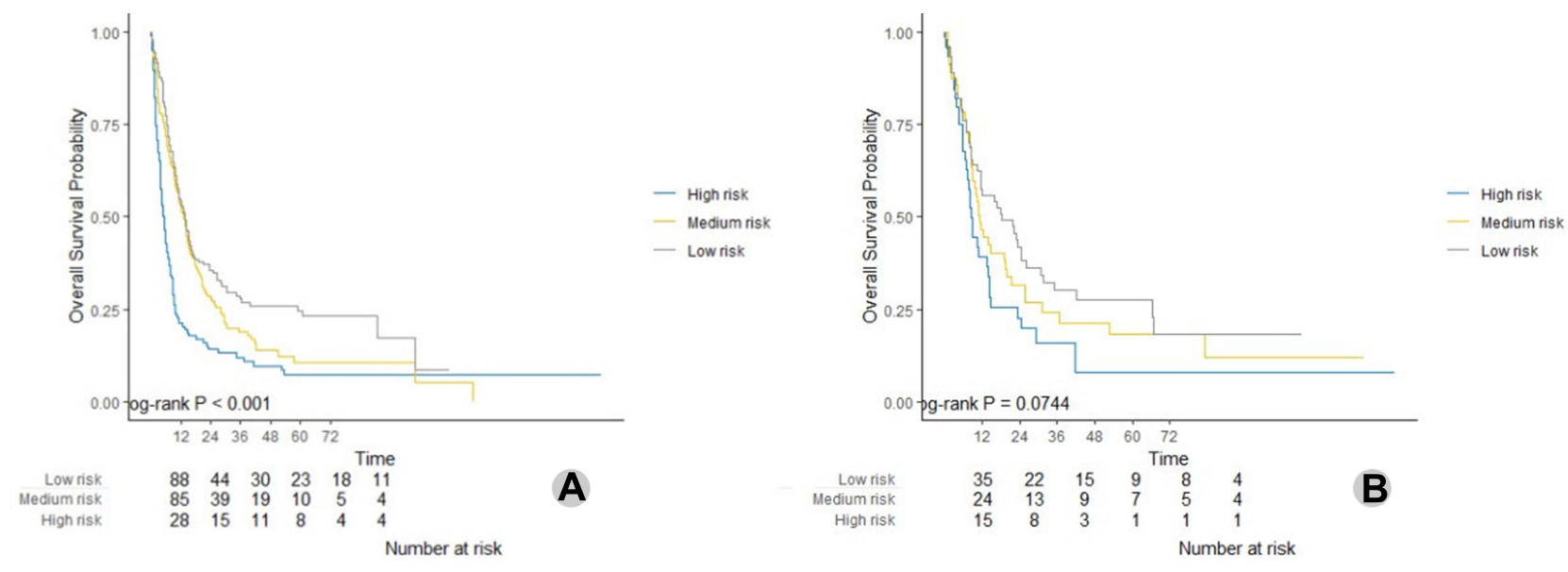

Figure 6 Kaplan-Meier survival curves for the training (A), and validation (B) groups.

angiogenesis through vascular endothelial growth factor, and up-regulate signaling pathways. ${ }^{29}$

Prognostic nomograms have been constructed and used to study the association between inflammatory markers, particularly NLR, on patients' prognosis and OS in many cancers, including gastric carcinoma, ${ }^{30}$ pancreatic neuroendocrine tumors, ${ }^{31}$ gallbladder cancer, ${ }^{32}$ colon cancer, ${ }^{33}$ breast cancer, ${ }^{34}$ and squamous cell carcinoma of the oral cavity. ${ }^{35}$ The variables we included in our established nomogram were NLR values and the presence of distant metastases at time of presentation. In addition to these variables, the previous studies concerning upper gastrointestinal tract tumors incorporated other variables in their nomogram, such as the TNM staging of patients, patients' age, gender, tumor size, and degree of differentiation. To our knowledge, our study was the first study to assess the relationship between NLR as a prognostic factor in cancers of the upper gastrointestinal tract collectively, and with an established nomogram to further increase the accuracy of prediction.

We suggest that upcoming research would further study the correlation between NLR and response to treatment, as the few published studies ${ }^{36,37}$ showed that NLR was a predictive marker of response to treatment in cancer patients. This may indicate that using inexpensive, readily available laboratory tests such as $\mathrm{CBC}$ can be used to predict the probability of distant metastases and accordingly be a major determinant in the management of upper gastrointestinal tract cancer patients, including more surveillance and probably better assignment of novel therapies.

We acknowledge some limitations in our study, such as the retrospective nature of the study design, as well as the potential selection bias given that all patients were sampled from the same cancer center, which might lead to referral bias. However, the value of NLR and other inflammatory parameters to predict prognosis and OS allows it to be 
potentially beneficial to the daily practice. Moreover, the absence of incorporation of patients' data on adjuvant chemotherapy in the three groups of patients in our study might have created a bias by affecting the OS. Further studies will be addressing the effect of adjuvant chemotherapy onpatient survival to eliminate such biases. We suggest that prospective multicenter studies to further validate our findings should be done. Also, additional prospective studies are needed to further elaborate on the role of NLR and other inflammatory markers as potential predictors of distant metastases and OS in cancer. We also suggest to further test the nomogram model that we used to ensure its' validity and practicality.

\section{Conclusion}

Our study concludes that location (ie, pancreatic, gastric and cholangiocarcinoma), high baseline NLR, along with other inflammatory markers are associated with distant metastases on presentation and poor median OS. The use of such simple and inexpensive laboratory tests from the patients' blood counts may further help in the utilization of this association in predicting prognosis in these patients. Further studies are warranted to evaluate the use of these markers in putting treatment and management plans for cancer patients.

\section{Acknowledgments}

The authors would like to acknowledge Mrs. Ayat Taqash and Dalia Rimawi for their help in constructing and interpreting the statistics.

\section{Disclosure}

The authors report no conflicts of interest in this work.

\section{References}

1. Balkwill F, Mantovani A. Inflammation and cancer: back to Virchow? Lancet. 2001;357(9255):539-545. doi:10.1016/S01406736(00)04046-0

2. Howlader N, Noone AM, Krapcho M, et al. SEER cancer statistics review; 1975-2017. National Cancer Institute, Bethesda, MD. Available from: https://seer.cancer.gov/csr/1975_2017/.

3. Gonzalez H, Hagerling C, Werb Z. Roles of the immune system in cancer: from tumor initiation to metastatic progression. Genes Dev. 2018;32(19-20):1267-1284. doi:10.1101/gad.314617.118

4. Coussens LM, Werb Z. Inflammation and cancer. Nature. 2002;420 (6917):860-867. doi:10.1038/nature01322

5. Liu J, Lin PC, Zhou BP. Inflammation fuels tumor progress and metastasis. Curr Pharm Des. 2015;21(21):3032-3040. doi:10.2174/ 1381612821666150514105741

6. van Horssen R, Ten Hagen TL, Eggermont AM. TNF-alpha in cancer treatment: molecular insights, antitumor effects, and clinical utility. Oncologist. 2006;11(4):397-408. doi:10.1634/theoncologist.11-4-397
7. Jana A, Krett NL, Guzman G, et al. NFkB is essential for activin-induced colorectal cancer migration via upregulation of PI3K-MDM2 pathway. Oncotarget. 2017;8(23):37377-37393. doi:10.18632/oncotarget. 16343

8. Laudisi F, Cherubini F, Monteleone G, Stolfi C. STAT3 interactors as potential therapeutic targets for cancer treatment. Int J Mol Sci. 2018;19(6):1787. doi:10.3390/ijms19061787

9. Geng SK, Fu SM, Fu YP, Zhang HW. Neutrophil to lymphocyte ratio is a prognostic factor for disease free survival in patients with breast cancer underwent curative resection. Medicine (Baltimore). 2018;97 (35):e11898. doi:10.1097/MD.0000000000011898

10. Kiriu T, Yamamoto M, Nagano T, et al. The time-series behavior of neutrophil-to-lymphocyte ratio is useful as a predictive marker in non-small cell lung cancer. PLoS One. 2018;13(2):e0193018. doi:10.1371/journal.pone.0193018

11. Şahin A, Toprak T, Kutluhan MA, Vural Y, Ürkmez A, Verit A. Increased neutrophil/lymphocyte ratio in testicular cancer. Arch Ital Urol Androl. 2019;91(2). doi:10.4081/aiua.2019.2.97

12. Chen G, Zhu L, Yang Y, Long Y, Li X, Wang Y. Prognostic role of neutrophil to lymphocyte ratio in ovarian cancer: a meta-analysis. Technol Cancer Res Treat. 2018;17:1533033818791500. doi:10.1177/ 1533033818791500

13. Uchinaka EI, Amisaki M, Yagyu T, et al. Prognostic significance of pre-surgical combined platelet count and neutrophil-lymphocyte ratio for patients with hepatocellular carcinoma. In Vivo (Brooklyn). 2019;33(6):2241-2248. doi:10.21873/invivo.11729

14. Guo D, Han A, Jing W, et al. Preoperative to postoperative change in neutrophil-to-lymphocyte ratio predict survival in colorectal cancer patients. Future Oncol. 2018;14(12):1187-1196. doi:10.2217/fon-2017-0659

15. Miyamoto R, Inagawa S, Sano N, Tadano S, Adachi S, Yamamoto M. The neutrophil-to-lymphocyte ratio (NLR) predicts short-term and long-term outcomes in gastric cancer patients. Eur J Surg Oncol. 2018;44(5):607-612. doi:10.1016/j.ejso.2018.02.003

16. Yodying H, Matsuda A, Miyashita M, et al. Prognostic significance of neutrophil-to-lymphocyte ratio and platelet-to-lymphocyte ratio in oncologic outcomes of esophageal cancer: a systematic review and meta-analysis. Ann Surg Oncol. 2016;23(2):646-654. doi:10.1245/ s10434-015-4869-5

17. Guthrie GJ, Charles KA, Roxburgh CS, Horgan PG, McMillan DC, Clarke SJ. The systemic inflammation-based neutrophil-lymphocyte ratio: experience in patients with cancer. Crit Rev Oncol Hematol. 2013;88(1):218-230. doi:10.1016/j. critrevonc.2013.03.010

18. Ohira M, Yoshizumi T, Yugawa K, et al. Association of inflammatory biomarkers with long-term outcomes after curative surgery for mass-forming intrahepatic cholangiocarcinoma. Surg Today. 2020;50(4):379-388. doi:10.1007/s00595-019-01905-7

19. Abu-Shawer M, Abu-Shawer O, Souleiman M, et al. Hematologic markers of lung metastasis in stage IV colorectal cancer. $J$ Gastrointest Cancer. 2019;50(3):428-433. doi:10.1007/s12029-018-0089-0

20. Grimes DA. The nomogram epidemic: resurgence of a medical relic. Ann Intern Med. 2008;149(4):273-275. doi:10.7326/0003-4819-1494-200808190-00010

21. Balachandran VP, Gonen M, Smith JJ, DeMatteo RP. Nomograms in oncology: more than meets the eye. Lancet Oncol. 2015;16(4):e173e180. doi:10.1016/S1470-2045(14)71116-7

22. Kattan MW, Hess KR, Amin MB, et al. American Joint Committee on Cancer acceptance criteria for inclusion of risk models for individualized prognosis in the practice of precision medicine. CA Cancer J Clin. 2016;66(5):370-374. doi:10.3322/caac.21339

23. Tham T, Machado R, Herman SW, Kraus D, Costantino P, Roche A. Personalized prognostication in head and neck cancer: a systematic review of nomograms according to the AJCC precision medicine core (PMC) criteria. Head Neck. 2019;41(8):2811-2822. doi:10.1002/ hed. 25778 
24. Sjoquist KM, Renfro LA, Simes RJ, et al. Personalizing survival predictions in advanced colorectal cancer: the ARCAD nomogram project. J Natl Cancer Inst. 2018;110(6):638-648. doi:10.1093/jnci/ $\mathrm{dj} \times 253$

25. Gilbride L, Siker M, Bovi J, Gore E, Schultz C, Hall WA. Current predictive indices and nomograms to enable personalization of radiation therapy for patients with secondary malignant neoplasms of the central nervous system: a review. Neurosurgery. 2018;82(5):595-603. doi:10.1093/neuros/nyx631

26. Chen JH, Zhai ET, Yuan YJ, et al. Systemic immune-inflammation index for predicting prognosis of colorectal cancer. World J Gastroenterol. 2017;23(34):6261-6272. doi:10.3748/wjg.v23.i34.6261

27. Wang K, Diao F, Ye Z, et al. Prognostic value of systemic immune-inflammation index in patients with gastric cancer. Chin J Cancer. 2017;36(1):75. doi:10.1186/s40880-017-0243-2

28. Kitamura T, Qian BZ, Pollard JW. Immune cell promotion of metastasis. Nat Rev Immunol. 2015;15(2):73-86. doi:10.1038/ nri3789

29. Galdiero MR, Garlanda C, Jaillon S, Marone G, Mantovani A. Tumor associated macrophages and neutrophils in tumor progression. $J$ Cell Physiol. 2013;228(7):1404-1412. doi:10.1002/jcp.24260

30. Choi JH, Suh YS, Choi Y, et al. Comprehensive analysis of the neutrophil-to-lymphocyte ratio for preoperative prognostic prediction nomogram in gastric cancer. World J Surg. 2018;42(8):2530-2541. doi:10.1007/s00268-018-4510-4

31. Tong Z, Liu L, Zheng Y, et al. Predictive value of preoperative peripheral blood neutrophil/lymphocyte ratio for lymph node metastasis in patients of resectable pancreatic neuroendocrine tumors: a nomogram-based study. World J Surg Oncol. 2017;15(1):108. doi:10.1186/s12957-017-1169-5
32. Deng Y, Zhang F, Yu X, Huo CL, Sun ZG, Wang S. Prognostic value of preoperative systemic inflammatory biomarkers in patients with gallbladder cancer and the establishment of a nomogram. Cancer Manag Res. 2019;11:9025-9035. doi:10.2147/CMAR.S218119

33. Chen L, Li Q, Wang Y, Yang J, Ma X. Prognostic value of nomogram based on pre-treatment inflammatory markers in patients with pulmonary-only synchronous metastases from colorectal cancer. Int Immunopharmacol. 2019;77:106001. doi:10.1016/j.intimp.20 19.106001

34. Cho U, Park HS, Im SY, et al. Prognostic value of systemic inflammatory markers and development of a nomogram in breast cancer. PLoS One. 2018;13(7):e0200936. doi:10.1371/journal.pone.0200936

35. Kao HK, Löfstrand J, Loh CY, et al. Nomogram based on albumin and neutrophil-to-lymphocyte ratio for predicting the prognosis of patients with oral cavity squamous cell carcinoma. Sci Rep. 2018;8 (1):13081. doi:10.1038/s41598-018-31498-z

36. Diem S, Schmid S, Krapf M, et al. Neutrophil-to-Lymphocyte ratio (NLR) and Platelet-to-Lymphocyte ratio (PLR) as prognostic markers in patients with non-small cell lung cancer (NSCLC) treated with nivolumab. Lung Cancer. 2017;111:176-181. doi:10.1016/j.lung can.2017.07.024

37. Graziano V, Grassadonia A, Iezzi L, et al. Combination of peripheral neutrophil-to-lymphocyte ratio and platelet-to-lymphocyte ratio is predictive of pathological complete response after neoadjuvant chemotherapy in breast cancer patients. Breast. 2019;44:33-38. doi:10.1016/j.breast.2018.12.014

\section{Publish your work in this journal}

Cancer Management and Research is an international, peer-reviewed open access journal focusing on cancer research and the optimal use of preventative and integrated treatment interventions to achieve improved outcomes, enhanced survival and quality of life for the cancer patient.
The manuscript management system is completely online and includes a very quick and fair peer-review system, which is all easy to use. Visit http://www.dovepress.com/testimonials.php to read real quotes from published authors. 